\title{
Nischarin regulates focal adhesion and Invadopodia formation in breast cancer cells
}

\author{
Mazvita Maziveyi', Shengli Dong1', Somesh Baranwal ${ }^{2}$ and Suresh K. Alahari ${ }^{1 *}$
}

\begin{abstract}
Background: During metastasis, tumor cells move through the tracks of extracellular matrix (ECM). Focal adhesions (FAs) are the protein complexes that link the cell cytoskeleton to the ECM and their presence is necessary for cell attachment. The tumor suppressor Nischarin interacts with a number of signaling proteins such as Integrin a5, PAK1, LIMK1, LKB1, and Rac1 to prevent cancer cell migration. Although previous findings have shown that Nischarin exerts this migratory inhibition by interacting with other proteins, the effects of these interactions on the entire FA machinery are unknown.
\end{abstract}

Methods: RT-PCR, Western Blotting, invadopodia assays, and immunofluorescence were used to examine FA gene expression and determine whether Nischarin affects cell attachment, as well as the proteins that regulate it.

Results: Our data show that Nischarin prevents cell migration and invasion by altering the expression of key focal adhesion proteins. Furthermore, we have found that Nischarin-expressing cells have reduced ability to attach the ECM, which in turn leads to a decrease in invadopodia-mediated matrix degradation.

Conclusions: These experiments demonstrate an important role of Nischarin in regulating cell attachment, which adds to our understanding of the early events of the metastatic process in breast cancer.

Keywords: Nischarin, Invasion, Invadopodia, Migration, Focal adhesion, Breast cancer, Integrins

\section{Background}

During metastasis, cancerous cells have to migrate through the extracellular matrix (ECM) for intravasation into blood and lymphatic vessels [1]. In order for this to occur, a cell must be able to detach from its original site and migrate through the breast stroma. The breast stroma, also known as the ECM, which is mostly composed of fat and matrix fibers while the rest is connective tissue [2]. This means a cell must be able to attach to ECM components through focal adhesions, as well as, degrade the ECM with matrix metalloproteinases. As the epithelial to mesenchymal (EMT) switch is in progress, the tumor has to rely on an altered microenvironment for migration [3]. Altered ECM composition and stiffness induce mammary malignancies [4-6]. This altered

\footnotetext{
* Correspondence: salaha@lsuhsc.edu

'Department of Biochemistry and Molecular Biology, LSUHSC School of Medicine, New Orleans, LA 70112, USA

Full list of author information is available at the end of the article
}

ECM is partly due to changes in fiber organization, and increased tension from cell-matrix adhesions [7, 8]. Since the ECM appears to be a key factor in breast cancer risk, it is important to study its regulation.

Nischarin is a cytosolic protein that binds to the cytosolic domain of integrin $\alpha 5$ to inhibit cell migration and invasion [9]. Nischarin interacts with Rac1, LIM Kinase (LIMK), Liver Kinase B1 (LKB1), p21 Protein (Cdc42/ Rac)-Activated Kinase 1 (PAK1), Rab14 and Insulin Receptor Substrates 1-4 (IRS1-4) [9-13]. Nischarin's location at chromosome 3p21.1 puts it in a category of genes that are associated with the development of many cancers. In vivo and in vitro studies from the Alahari lab have classified Nischarin as a tumor suppressor because it inhibits tumor progression and metastasis [11]. Since mutations in Nischarin are related to reduced patient survival [11], our goal is to understand how Nischarin affects cell interaction with the ECM. This study has determined that Nisch decreases the expression of several 
FA proteins, including Integrins. Furthermore, we have discovered that this aberrant cell attachment translates into an inability of these cells to form invadopodia and secrete matrix metalloproteinases. Since this work explains the role of Nischarin in the early stages of the metastatic process, the results of our research will contribute to our understanding of the tumor microenvironment. Understanding the role Nischarin and other tumor suppressors play in the prevention of breast cancer cell survival and migration will provide more knowledge for novel breast cancer therapies.

\section{Methods}

\section{Cell culture}

Human breast cancer MDA-MB-231, MDA-MB-231 Nischarin, MCF7 shScramble, MCF7 GFP Nisch, MCF7 shNisch, WT-Nischarin mouse embryonic fibroblasts (MEFs), HET-Nischarin MEFs, Null Nischarin MEFs, HEK293, Cos7, and NIH3T3 cells were grown in Dulbecco's Modified Eagle Medium (DMEM) at $37{ }^{\circ} \mathrm{C}, 5 \% \mathrm{CO}_{2}$ supplemented with $10 \%$ fetal bovine serum and $1 \%$ penicillin/streptomycin (Gibco, Waltham, MA). MCF10A cells were grown in DMEM/F12 media supplemented with $10 \%$ fetal bovine serum and $1 \%$ penicillin/streptomycin (Gibco, Waltham, MA). For wound healing assays the cells were seeded on a 6-well plate and allowed to attach for $12 \mathrm{~h}$. Then, a wound was created with a $200 \mu \mathrm{l}$ tip and imaging was done at various time points. Percentage of wound closure was obtained using Image J. For live cell imaging, different subsets of 10,000 MDA-MB-231 cells were plated onto gelatin-coated $35-\mathrm{mm}$ glass bottom dishes (2D) (MatTek) or NIH3T3 fibroblast-derived matrices (3D) overnight. The cells were cultured at $37^{\circ} \mathrm{C}$ with 5\% CO2 using a Live Cell Environmental Chamber (NEUE Group, Ontario, NY). Five random acquisition points were pre-determined for each experimental well, each pre-set location was photographed every $10 \mathrm{~min}$ for a period of $10 \mathrm{~h}$ using an Olympus IX71 microscope. Cell position and average speed were determined and calculated using Slidebook software.

\section{Tissues used}

Twenty human breast cancer surgical specimens, thirteen malignant and seven non-cancerous tissues were obtained as frozen tissue sections from the Southern Division (Birmingham, AL), Eastern Division (Philadelphia, PA), and Mid-Western Division (Columbus, $\mathrm{OH}$ ) of the Cooperative Human Tissue Network.

\section{Real-time PCR}

Total RNA was isolated from cultured cells with TRIzol reagent (Invitrogen, Carlsband, CA). cDNA was generated from $2 \mu \mathrm{g}$ of RNA using the Invitrogen/Applied Biosystems/ABl High Capacity cDNA Reverse Transcriptase
Kit (Carlsband, CA). For gel detection, samples were run on a 3\% agarose gel. Quantitative real-time PCR (qRTPCR) was performed with $2 \times$ SYBR green mix (Roche, Basel, Switzerland) according to the manufacturer's instructions. $\Delta$ Ct was calculated as $\mathrm{Ct}$ (sample)-Ct (Bactin or GAPDH). Relative expression was calculated as $\Delta \Delta \mathrm{Cq}$ $\left(2^{\wedge}-\Delta \mathrm{Ct}\left(\right.\right.$ sample) $/ 2^{\wedge}-\Delta \mathrm{Ct}$ (control cell line).

\section{Transwell invasion assay}

The invasion assays were performed using Tanswell invasion chambers (Corning, Corning, NY) coated with $10 \mu \mathrm{g} / \mathrm{ml}$ Fibronectin (bottom) and matrigel (top). MCF10A cells were transferred to the top of the chamber with the indicated media. After incubation for $24 \mathrm{~h}$ at $37{ }^{\circ} \mathrm{C}$ in an atmosphere containing $5 \% \mathrm{CO}_{2}$, invaded cells on the lower surface were stained with crystal violet stain and counted under a light microscope.

\section{Coimmunoprecipitation}

For Nischarin-Cortactin binding experiments, HEK293 cells were transiently transfected with $4 \mu \mathrm{g}$ of FlagCortactin and Myc-Nischarin using Lipofectamine ${ }^{\bullet}$ Reagent 2000 (Invitrogen, Carlsband, CA). As a control, $3 \mu \mathrm{g}$ Myc- $\beta$ gal was cotransfected with Flag-Cortactin. Forty-eight hours later, cells were lysed in lysis buffer (50 mM Tris, pH 7.5, 0.1\% Triton X-100, 0.3 M sucrose, $100 \mathrm{mM} \mathrm{KCl}$, and $1 \mathrm{mM} \mathrm{CaCl}$ ) with protease inhibitors ( $1 \mathrm{mM}$ PMSF, $2 \mu \mathrm{g} / \mathrm{ml}$ aprotinin, and $5 \mu \mathrm{g} / \mathrm{ml}$ leupeptin). The lysates were immunoprecipitated with appropriate antibodies and pulled down by Protein G Sepharose beads (Uppsala, Sweden). Proteins were then immunoblotted for Myc and Flag antibodies.

\section{Antibodies}

Antibodies and dilutions were used as follows: mouse monoclonal anti-Nischarin (BD Biosciences, San Diego, CA; 1:1000), rabbit polyclonal anti-human Fibronectin (Sigma, St. Louis, MO; 1:1000), rabbit polyclonal antiCortactin (Santa Cruz, Dallas, TX; 1:3000), rat anti-Flag (Stratagene, San Diego, CA; 1:5000), mouse monoclonal anti-human C-myc (BioXCell, West Lebanon, NH; 1:2500), mouse monoclonal anti-Paxillin (BD Biosciences, San Diego, CA; 1:5000), mouse monoclonal antiHIC5 (BD Biosciences, San Diego, CA; 1:5000), mouse monoclonal anti-CRP1 (BD Biosciences, San Diego, CA; 1:1000), mouse monoclonal anti-MCAM (BD Biosciences, San Diego, CA; 1:1000), mouse monoclonal antiZyxin (Santa Cruz, Dallas, TX; 1:100), rabbit polyclonal anti-VASP (Sigma, St. Louis, MO; 1:100), rabbit polyclonal anti-Integrin $\alpha 5$ (EMD Millipore, Billerica, MA; 1:100) and mouse monoclonal anti-Vinculin (Sigma, St. Louis, MO; 1:5000). The SNAKA51 antibodies (1:100) were a gift from Kenneth Yamada. 


\section{Fibroblast matrices}

To prepare coverslips for matrix deposition, $2 \mathrm{ml}$ of $0.2 \%$ gelatin was added to the coverslips and incubated for $1 \mathrm{~h}$ at $37{ }^{\circ} \mathrm{C}$. The gelatin was aspirated and $2 \mathrm{ml}$ of cold PBS was added to wash the coverslip. After aspiration of the PBS, $2 \mathrm{ml}$ of $1 \%$ glutaraldehyde was added and incubated at $37{ }^{\circ} \mathrm{C}$ for $30 \mathrm{~min}$. The coverslips were washed three times for $5 \mathrm{~min}$ each with cold PBS. $2 \mathrm{ml}$ of $1 \mathrm{M}$ ethanolamine was then added and incubated for $30 \mathrm{~min}$ at room temperature. The three PBS washes were repeated again to remove all traces of ethanolamine. $2 \mathrm{ml}$ of matrix media (DMEM $+10 \% \mathrm{FBS}+\mathrm{P} / \mathrm{S}$ + Anti-Anti $+50 \mu \mathrm{g} / \mathrm{ml}$ ascorbic acid) was then added. Cells were plated on the coverslips in $2 \mathrm{ml}$ of matrix media. These cells were cultured for $24 \mathrm{~h}$. After $24 \mathrm{~h}$, the medium was aspirated and replaced with fresh matrix medium every day for six days. On day 6 , the medium was carefully aspirated and the matrix was decellularized. Curvelet transform plus FIRE (CT-FIRE) algorithm was used to analyze fiber width and number.

\section{Fluorescent-gelatin degradation assay}

Coverslips were cleaned with $20 \%$ nitric acid and coated with poly-L-lysine in a 24 -well plate. The poly-L-lysine was fixed with $0.5 \%$ glutaraldehyde before adding the Oregon green Gelatin (Life Technologies, Calsbad, CA). Residual reactive groups were quenched by sodium borohydride. $4 \times 10^{3}$ cells were plated and incubated at $37^{\circ} \mathrm{C}$. Images were visualized by confocal microscopy.

\section{Statistical analysis}

All statistical analyses were performed using Graphpad Prism 5.0 software (San Diego, CA) using a nonparametric two-tailed $t$ test or one way anova.

\section{Results}

Nischarin alters the expression of focal adhesion proteins The tumor suppressor Nischarin has previously been shown to inhibit cell migration in breast cancer cells [14]. To confirm that Nischarin prevents cancer cell migration, we performed a time course wound healing assay with our previously published MDA-MB-231 and MDA-MB-231 Nischarin cells [11] for up to $24 \mathrm{~h}$ (Fig. 1a). Quantitation of the percentage of wound closure at $0,3,6,9,12$, and $24 \mathrm{~h}$ revealed that MDA-MB231 Nischarin cells have an overall reduced cell migration and the effect is more robust at 9 and $12 \mathrm{~h}$ (Fig. 1b). To further validate these findings, we explored whether Nischarin is still able to slow down the migration of cells attached in a 3D environment. We tracked live cell migration of cells seeded on a thin $2 \mathrm{D}$ gelatin layer or $3 \mathrm{D}$ fibroblast-derived matrices for a period of ten hours (Fig. 1c). MDA-MB-231 Nisch cells had a reduction in average speed on both $2 \mathrm{D}$ and $3 \mathrm{D}$ environments when compared to 231 cells (Fig. 1d). These experiments confirm our previously published work showing that Nischarin reduces cell migration.

Although previous findings showed that Nischarin exerts this migratory inhibition by interacting with other proteins, such as LIMK and PAK1 [11], the effects of these interactions on the entire FA machinery are unknown. FAs are the protein complexes that link the cell cytoskeleton to the ECM and their presence is necessary for cell attachment [15]. Tyrosine phosphorylation is a key event that occurs prior to the recruitment of other FA proteins [15]. To assess the effects of Nischarin on the FA machinery, we performed qRT-PCR and Western Blots of twelve key focal adhesion proteins. Out of twelve, we found five genes with consistently significant decreases in expression (Fig. 2). Paxillin is one of the first proteins to arrive at a nascent focal adhesion site [16]. Nischarin-expressing MDA-MB-231 and MCF7 cells have a significant reduction of Paxillin RNA and protein compared to Nisch-lacking cells (Fig. 2a-c). The ability of FA proteins to form protein-protein interactions is necessary for the formation of stable FAs. HIC-5 is a Paxillin-related protein that coordinates multiple protein-protein interactions in the early stages of FA development [15, 17]. MDA-MB-231 and MCF7 cells expressing Nisch have significantly less HIC-5 RNA and protein when compared to MDAMB-231 cells (Fig. 2a-c).

In the intermediate stages of FA development, Actin filament bundling must occur in order for there to be a stable protrusion. Cysteine-rich protein 1 (CRP1) is a protein that regulates Actin filament bundling by binding to the Actin-bundling protein $\alpha$-Actinin [18]. We also found reduced expression of these proteins in the presence of Nischarin (Fig. 2a-c). Finally, we assessed the expression of the Melanoma Cell Adhesion Molecule (MCAM), also known as, CD146, which is considered a marker for mesenchymal cells [19]. Nisch-expressing cells had a reduction in MCAM expression (Fig. 2a-c). To determine whether this decrease of FA protein expression is specific to cancer cells, we transiently transfected Cos7 cells (monkey kidney) with Myc or MycNischarin and examined expression of the proteins. The presence of Nisch did not alter expression of any one of the FA proteins in Cos7 cells but only in the MDA-MB231 cancer cells (Fig. 2d). These data demonstrate that the presence of Nischarin leads to a decrease in the expression of some FA proteins in cancer cells only.

\section{Nischarin alters integrin expression}

Zyxin is a protein that concentrates along the Actin cytoskeleton and localizes at FA sites [20]. We previously determined the number of FAs in Nisch positive 


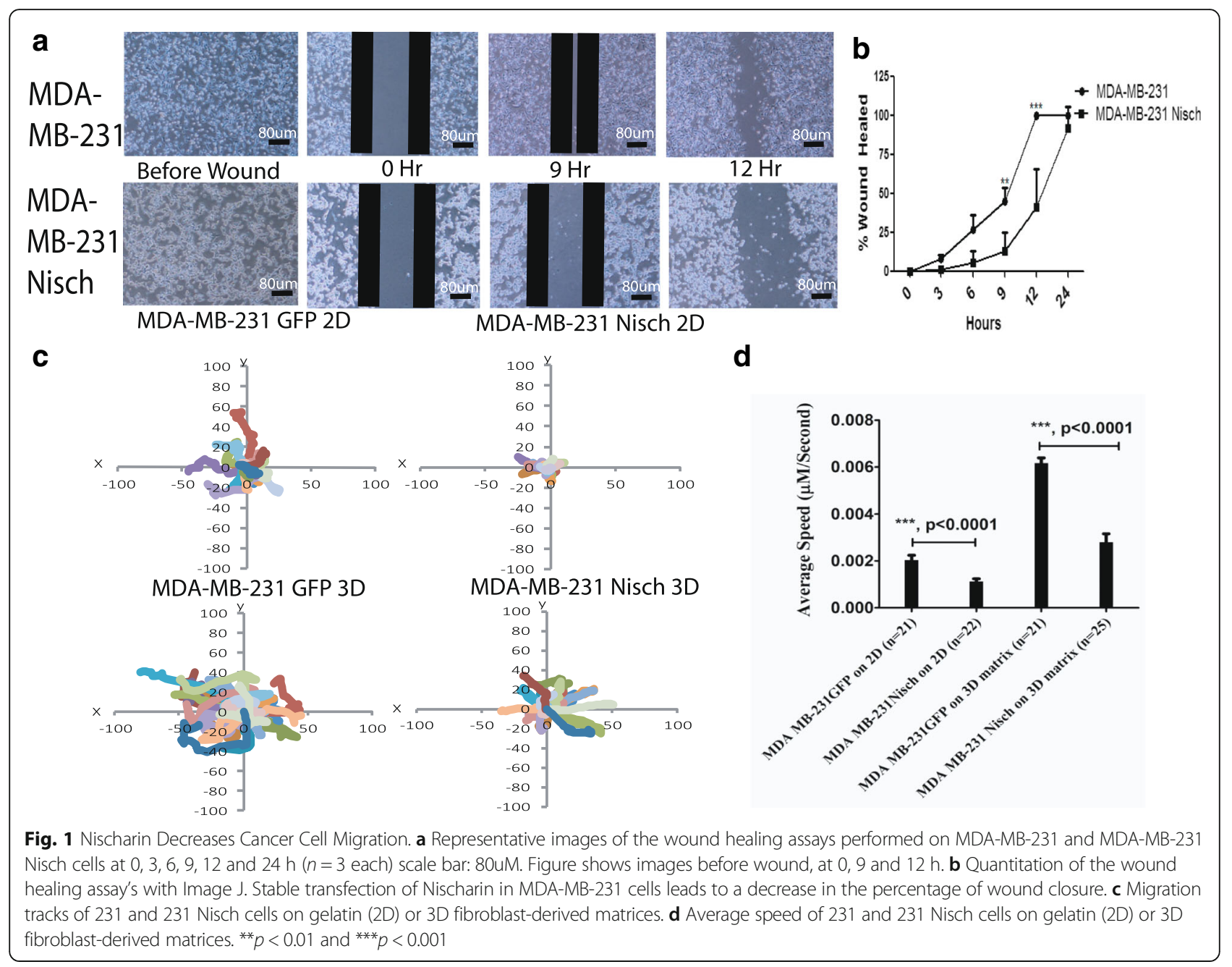

and negative cells. Mouse embryonic fibroblasts (MEFs) are widely used for quality visualization of FAs due to their complete mesenchymal nature. We stained WT and Null Nisch MEFs [21] with Zyxin and quantitated the number of FAs using CellProfiler. Null Nischarin MEFs had an increase in the number of FAs and percentage of area covered by FAs (Fig. 3a). These quantitated images support the data in Fig. 1 that showed the decrease of migration and FA protein expression in Nischarin-expressing cells.

The Ena/VASP family of proteins play an important role in cell migration, invasion and adhesion [22]. In fact, they are capable of binding the Integrin $\alpha 5$ (ITGA5) cytoplasmic tail similar to Nischarin [22, 23]. We examined VASP protein expression and found that its expression is reduced in Nischarin-expressing MDA-MB-231 cells (Fig. 3b). We determined that this is cancer cell specific since addition of Nisch to Cos7 cells does not affect VASP expression (Fig. 3c). To further assess the impact of Nischarin on the number of VASP-positive FAs, we stained MDA-MB-231 and MDA-MB-231 Nisch cells with VASP and used the CellProfiler software to count the number of VASP FAs per cell (Fig. 3d). MDAMB-231 Nisch cells had a reduction in the number of VASP FAs and percentage of area covered by VASP positive FAs. To determine whether we see the same pattern in WT and Null Nischarin MEFs, we stained them with VASP and quantitated FA numbers using Cell Profiler. Null Nischarin MEFs had an increase in the number of VASP FAs and percentage of area covered by VASP positive FAs (Additional file 1: Fig. S1). Since Nisch and VASP both bind to Integrins, we next determined whether Nischarin binds to VASP to reduce its presence in FAs. Co-Immunoprecipitation experiments between Nischarin and VASP showed that there is no interaction between the two proteins (Additional file 1: Fig. S1B). These results demonstrate that while Nischarin and VASP both bind ITGA5, they do not interact but the presence of Nisch leads to a decrease in VASP-positive FAs.

We have established that Nisch regulates FA protein expression. Integrins are membrane proteins that 


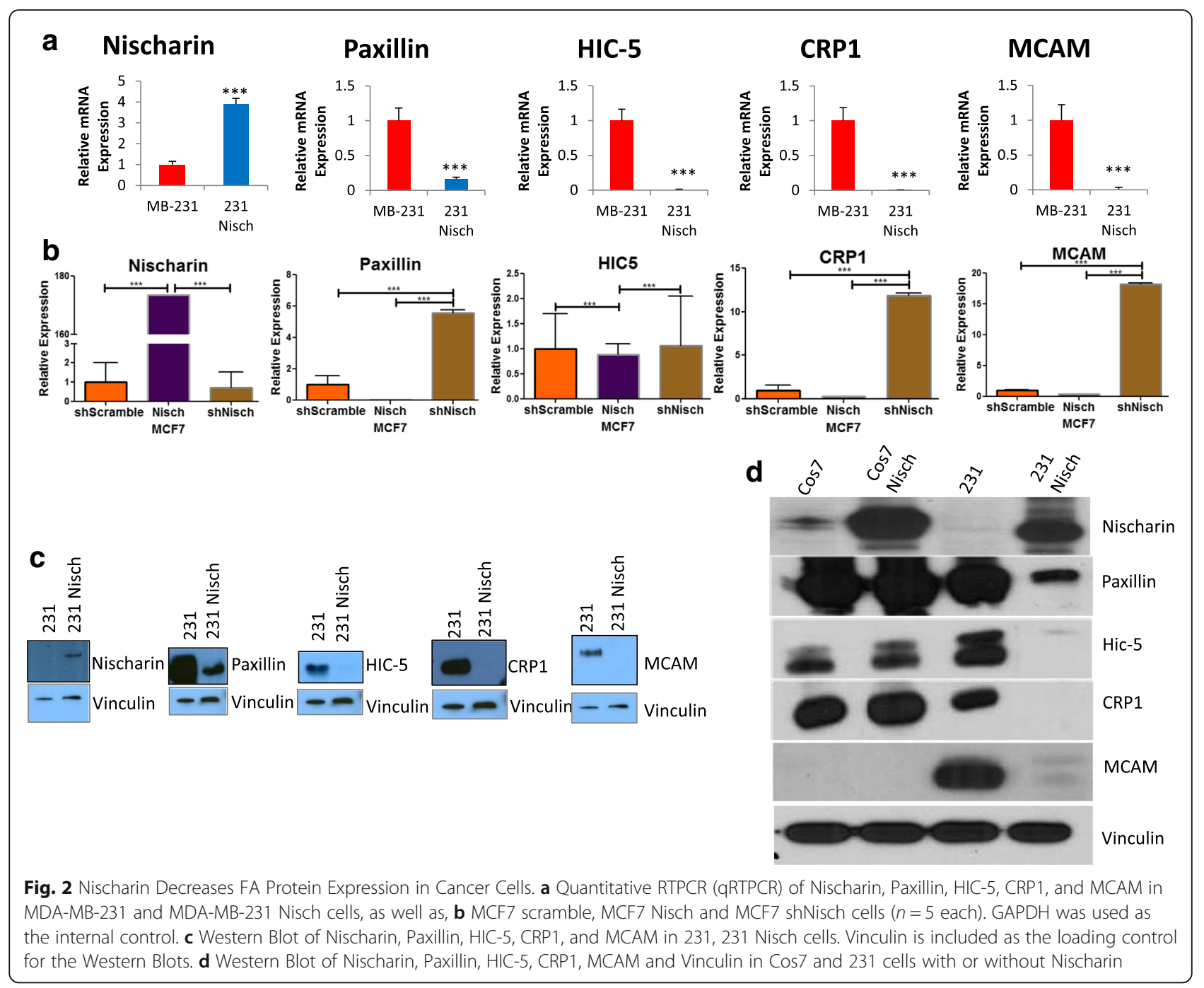

connect the FA protein complexes to the extracellular matrix (ECM) [15]. We have previously shown that Nischarin binds to and inhibits the expression of ITGA5 [23]. To support our previous findings, we visualized whether this Nisch-mediated ITGA5 reduction is still present in FA sites. Vinculin is often used as a marker to detect Integrin-mediated cell-matrix adhesions by immunofluorescence [24, 25]. Next, to determine whether Nischarin inhibits targeting of ITGA5 to FA sites, we stained WT and Null Nisch MEFs with Vinculin (green) and ITGA5 (red) (Fig. 3e). Merged images of Vinculin and ITGA5 showed less colocalization of ITGA5 with FAs in WT MEFs when compared to Null MEFs (Fig. 3e). We further validated the expression of ITGA5 and activation of its regulator, FAK. Western Blotting demonstrated an increase in protein expression of ITGA5 and pFAK in Null Nisch MEFs (Fig. 3f). Cancer cells exhibit increased autophosphorylation of FAK at Y397 [26].
This activated FAK is responsible for initiating the FA signaling pathway, thus allowing Integrin activation. We have established that Nisch inhibits ITGA5 expression, and thus the whole Integrin $\alpha 5 \beta 1$ complex is reduced [11]. For the Nisch blot, the lower band present in Null MEFs reflects the 172 amino acid deletion that produces a Null Nisch phenotype [21]. The doublet band for HET MEFs reflects the normal allele (top) and the truncated allele (bottom) [21].

Furthermore, we identified the levels of active Integrin $\alpha 5 \beta 1$ by immunofluorescence using the conformationdependent ITGA5 antibody SNAKA51 [27]. There was significantly less SNAKA51 fluorescence in 231 Nis cells, indicating less active ITGA5 (Fig. 3g). This is the first time we have been able to quantify the reduction in the amount active Integrin $\alpha 5 \beta 1$.

Finally, we assessed whether Nischarin alters the expression of other Integrins. We performed RT-PCR experiments that showed alterations in the expression of 


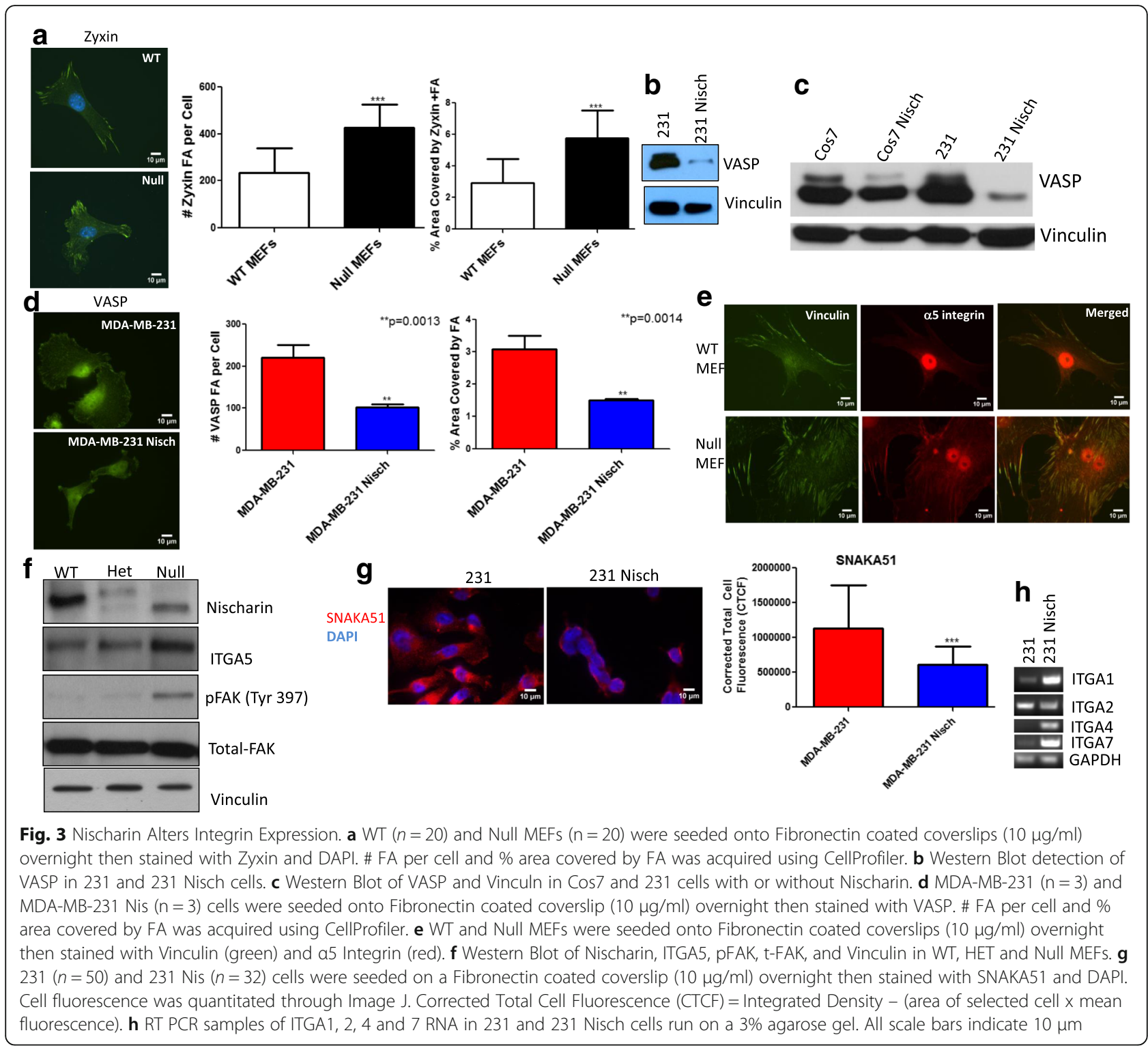

four other Integrins in the presence of Nischarin. Integrins $\alpha 1,4$, and 7 levels were increased, while Integrin $\alpha 2$ was decreased in the presence of Nischarin (Fig. 3h). Taken together, we have established that Nischarin regulates the expression of multiple $\alpha$-Integrins.

\section{Nischarin regulates Invadopodia}

Integrins also stabilize invadopodia, which are protrusive cell-matrix adhesions that contain proteases for ECM degradation. In addition to proteases, these protrusions are enriched with proteins including, Cortactin, Tks4 and Tks5 [28]. To determine whether Nischarin affects invadopodia formation, we performed a gelatin degradation assay [29]. To compare degradative patterns between MDA-MB-231 and MDA-MB-231 Nischarin cells, we plated the cells on fluorescent gelatin-coated coverslips and allowed them to degrade the gelatin matrix. A greater number of MDA-MB-231 cells degraded the fluorescent substrate at both four and five hours of time (Fig. 4a-b). To determine whether this degradation also occurs on Fibronectin fibers, we stimulated NIH3T3 fibroblasts to produce Fibronectin-derived matrix (FDM). After decellularization, we plated WTNisch and Null-Nisch MEFs on the NIH3T3 FDMs to visualize matrix alterations. Seeding Null MEFs on the FDMs reduced the Fibronectin signal (Fig. 4c), suggesting that the Null MEFs have degraded the matrix. Taken together, Nischarin negative (Null-Nisch) cells degrade the ECM at a greater rate than WT cells.

To further asses this in vitro while still maintaining a physiologically realistic culture substrate, we generated fibroblast-derived Fibronectin-rich matrices from 


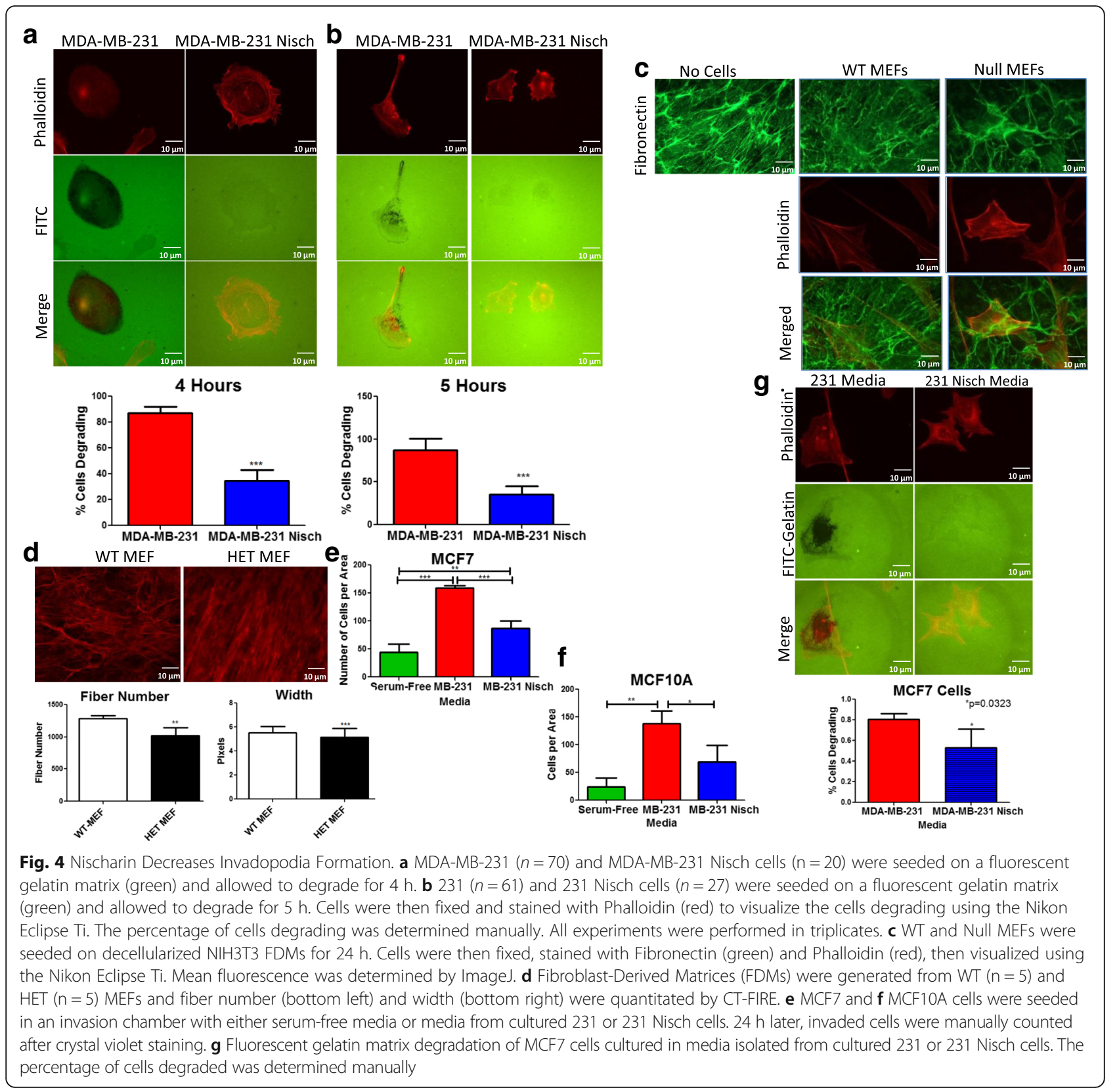

WT-Nisch and HET-Nisch MEFs. Fibroblasts were cultured for eight days in the presence of ascorbic acid to stimulate matrix production. At day 8 , cells were decellularized and Fibronectin was detected on the fibers by confocal microscopy (Fig. 4d). WT-Nisch and HET-Nisch fibronectin fibers were quantitated by CT-FIRE and the analysis revealed that WT-Nisch fibers have a greater fiber number and fiber width than the Het-Nisch fibers (Fig. 4d). These data suggest that the HET cells that were seeded on the substrate were degrading the matrix.

Since the invadopodia are enriched with several secreted proteins, we explored the effects of secreted media from MDA-MB-231 and MDA-MB-231 Nisch cells on the invasion of moderately invasive MCF7 cells. Media from MDA-MB-231 cells increased the invasiveness of MCF7 cells, while addition of media from MDAMB-231 Nisch cells reduced the percentage of MCF7 cells degrading the fluorescent gelatin matrix (Fig. 4e). To further explore this on non-invasive cells, we isolated media from MDA-MB-231 and MDA-MB-231 Nisch cells and cultured them with normal MCF10A cells to monitor invasion. MCF10A cells are normal, noncancerous breast epithelial cells that typically do not have the capacity to invade. Adding media from MDA-MB-231 cells significantly induced the invasiveness of MCF10A cells in transwell inserts (Fig. 4f). Culturing the cells with 
media from MDA-MB-231 Nisch cells significantly reduced the invasive capability of the cells. Next, we wanted to determine whether media from MDA-MB-231 Nisch cells reduced invadopodia formation. Similar to the transwell invasion assays, MCF7 cells cultured in 231 Nisch media had a reduction in the percentage of cells degrading the gelatin matrix (Fig. 4g). Taken together, these results suggest that Nisch prevents invadopodia formation, and media isolated from Nisch expressing cancer cells might have an altered protease profile.

\section{Nischarin decreases the expression of Invadopodia proteins}

Cortactin has been shown to be good marker for invadopodia [30] and its mRNA expression is increased in malignant human breast tissues (Fig. 5a). We investigated whether Nisch regulates formation of invadopodium through Cortactin. QRT-PCR experiments showed no significant difference in the expression of Cortactin mRNA in MDA-MB-231 versus MDA-MB-231 Nisch cells (Fig. 5b). Agarose gel visualization of Cortactin cDNA from MDA-MB-231 and MDA-MB-231 Nisch cells confirmed our findings of no significant differences (Fig. 5c). Western blot detection of Cortactin also confirmed that the protein expression was not altered by the presence of Nisch (Fig. 5d). Since we did not find any changes in Cortactin RNA or protein expression, we thought Nischarin might alter invadopodia formation by interacting with Cortactin. Coimmunoprecipitation experiments demonstrated that Nischarin does not interact with Cortactin (Fig. 5e). These data suggest that Nischarin uses another mechanism to prevent invadopodia formation.

Tks4 and Tks5 are adaptor proteins that are required for invadopodia formation [31]. RT-PCR of Tks4 and Tks5 showed a decrease in the expression of Tks4, but not Tks5 (Fig. 5f). During invadopodia formation, Tks5 recruits AFAP-110 to the future protrusion site [32]. We found decreased amounts of AFAP-110 in Nisch expression cells (Fig. 5g). Although the expression of, Cortactin, is not regulated by Nischarin, other invadopodium proteins seem to be affected.

Fourteen Matrix-Metallo Proteases (MMPs) have been noted to have increased expression in breast cancer tissue when compared to normal breast tissue [33]. To assess whether the presence of Nischarin in breast cancer cells affects MMPs, we performed reverse transcriptase PCR of several MMPs on MDAMB-231 and MDA-MB-231 Nisch cells. We found reduced expression of MMPs 1, 2 and 9 in the Nischarin expressing cancer cells (Fig. 5h). Taken together, our findings suggest that the presence of Nischarin in breast cancer cells leads to a down regulation of MMPs 1, 2, and 9, which leads to a reduction in ECM degradation.
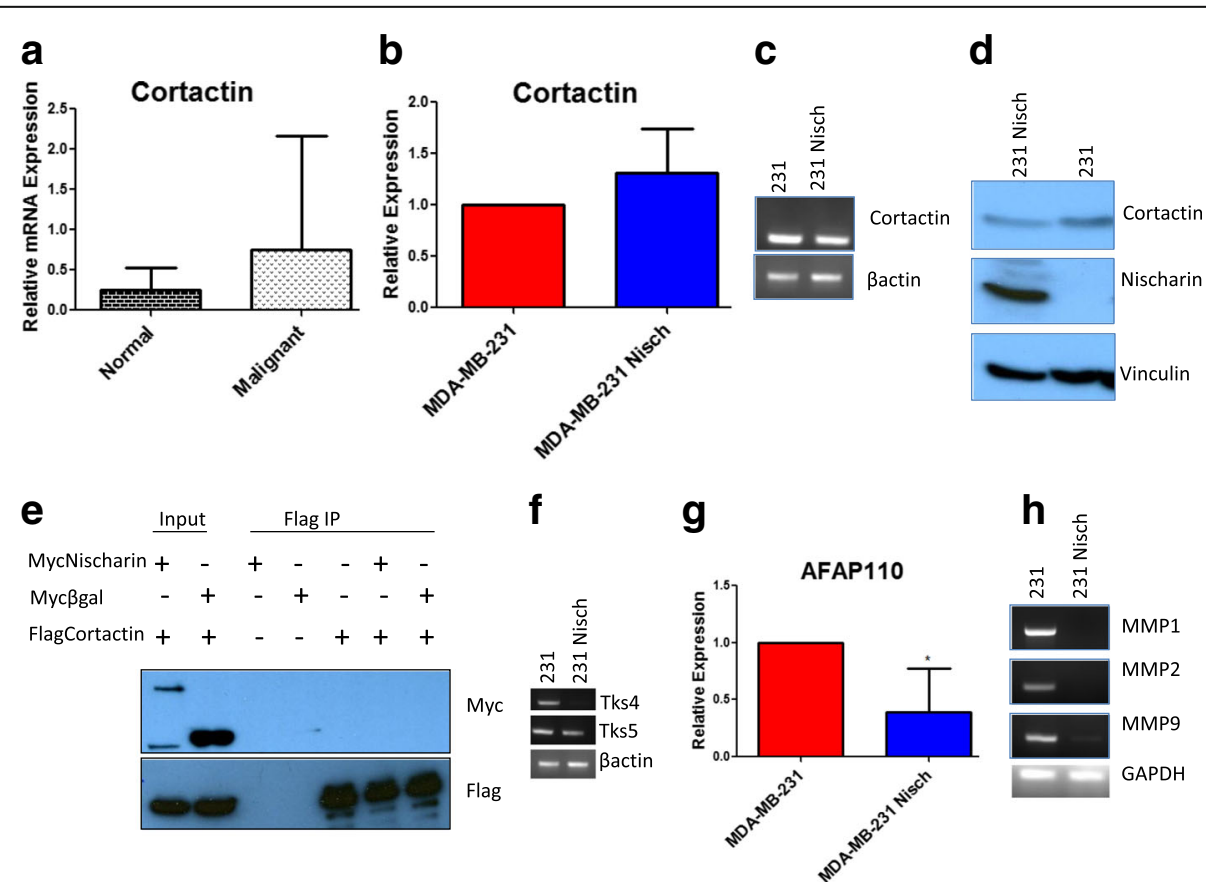

Fig. 5 Nischarin Decreases the Expression of Invadopodia Proteins. a qRT-PCR of Cortactin in normal $(n=7)$ and malignant $(n=13)$ human breast samples. $\mathbf{b}$ qRTPCR of Cortactin in $231(n=8)$ and 231 Nischarin $(n=8)$ cells. $\mathbf{c}$ RT PCR of Cortactin RNA in 231 and 231 Nisch cells. d Western Blot of Cortactin protein expression in 231 and 231 Nisch cells. Vinculin is shown as a control. e Co-Immunoprecipitation of Myc-Nischarin and FlagCortactin in HEK 293 cells. $\mathbf{f}$ RT-PCR of Tks4 and Tks5 RNA in 231 and 231 Nisch cells run on a 3\% agarose gel. $\mathbf{g}$ qRTPCR of AFAP110 in 231 ( $n=6$ ) and 231 Nisch $(n=8)$ cells. $\mathbf{h}$ RT PCR of MMPs 1, 2 and 9 RNA in 231 and 231 Nisch cells run on a 3\% agarose gel. ${ }^{*} p<0.05,{ }^{* *} p<0.01$ 


\section{Discussion}

Epithelial-mesenchymal transition (EMT) is an important step during the process of cancer cell detachment from the primary site to the distant organs [34]. Tumor cells then use the fibril and glycoprotein rich ECM for migration and cell adhesion. Our data show the ability of the tumor suppressor, Nischarin to regulate cell attachment to the ECM. Although we have previously shown that Nisch prevents cell migration [11, 14, 23], we have not determined the exact mechanism. We confirmed that Nisch prevents cancer cell migration since we see a reduction in the percentage of wound healing.

The results from our micrographs were consistent with previous wound healing assays performed in Nisch positive and negative MDA-MB-231 cells [35]. Many cell migration assays, including the wound healing assay, are on 2D and do not take into account the complexity of the solid tumor. Since cancer cells in a solid tumor are in a three-dimensional environment, we determined Nischarin's ability to reduce the migration of cells in 3D matrix. We identified that Nischarin was able to reduce the migration of cancer cells on 3D matrices. As cell migrates through a $3 \mathrm{D}$ environment, it experiences resistance from the ECM that could alter attachment, polarization and migration [36]. Of great importance to us is the effect that the 3D ECM has on FA proteins.

To determine whether Nisch regulates cell-matrix attachments, we performed a screen of key FA proteins using MDA-MB-231 and MCF7 cells. Although some proteins did not have a change in expression, VASP, Paxillin, HIC-5, CRP1 and MCAM have significant differences in RNA and protein expression between Nischarin expressors and non-expressors. Reduction in FAs number per cell could possibly be due to the changes in these proteins expression. More importantly, we detected the changes were only specific to cancer cells (Fig. 6). Highly motile and invasive cancer cells already exhibit expression of oncogenic proteins that crosstalk with the FA signaling pathway. For example, cancer cells have an increase in FAK and Src activation, which contributes to increased FA protein recruitment and phosphorylation [37, 38]. This explains why introducing Nisch into non-cancerous Cos7 cells does not alter FA protein expression. Previous studies have shown that Nisch does not co-localize to FA sites [23]. No other studies have been able to explain how Nischarin reduces the expression of ITGA5. Since there is bidirectional crosstalk between Integrins and FA proteins, it is possible that the down regulation of Integrins leads to a down regulation of FA proteins. In other words, if there is no need for the proteins to be in FA machinery due to the unavailability of Integrins, and thus the expression of FA proteins is reduced in cancer cells.

The FA proteins form the macromolecular focal adhesion unit that helps attaching the cells to the ECM. Integrins are the proteins that actually connect
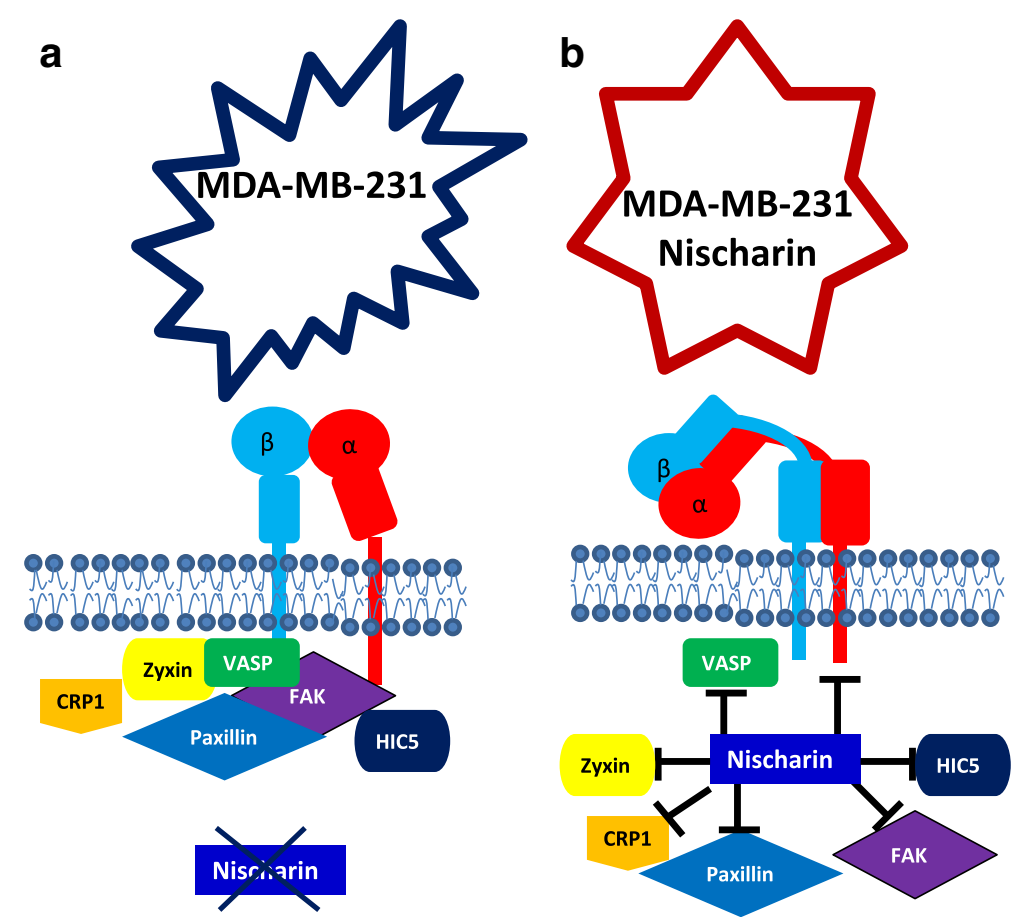

Fig. 6 Nischarin Decreases FA Expression in Breast Cancer Cells. a 231 cells are able to have more FA protrusions due to the activation of the FA interactome. $\mathbf{b}$ The presence of Nisch in 231 cells inhibits the expression of key FA proteins, which results in fewer protrusions 
the FA complexes to the ECM. We were able to see that the absence of Nisch increases the targeting of $\alpha 5$-Integrin to FAs (Fig. 6). Furthermore, we noticed a reduction in the amount of active $\alpha 5$-Integrin in Nisch-expressing breast cancer cells, suggesting a possible reason for reduction of FAs in Nisch expressing cells $[11,23]$.

Integrin $\alpha 5 \beta 1$ participates in the adhesion necessary for invadopodia formation [39], and thus it was necessary to analyze cell invasion. We have previously published cell invasion data demonstrating the ability of Nisch to reduce invasion on Fibronectin transwell inserts [11]. Our current findings showed a decrease in fluorescent matrix degradation of breast cancer cells in the presence of Nischarin. Furthermore, a greater amount of FDM degradation was seen on NIH3T3 matrices seeded with Null Nischarin MEF cells. Similarly, matrices produced from Nischarin HET MEF cells had a reduction of fiber number and width, likely due to the invasive capacity of cells with reduced Nisch expression. Transwell and gelatin degradation experiments of cell supernatants revealed that Nischarin positive cancer cells prevent degradation. The increased matrix degradation seen in cells lacking Nischarin explains why we see a reduction in fiber number and width. Our results agree with results from other groups that have shown that the formation of invadopodia protrusions correlates with ECM degradation [40].

Since we see more degradation in the Nischarin Null cells, we checked the expression of key invadopodium machinery. There was no statistical difference in Cortactin expression between Nischarin positive and negative breast cancer cells. Furthermore, Nischarin did not interact with Cortactin, the key regulator of invadopodia. We did, however, find a reduction in the key invadopodia proteins Tks4 and AFAP-110. Assessment of MMP1, 2 , and 9 in the cells showed that Nischarin positive cancer cells have a reduction in expression of all three MMPs. Other studies have shown that the expression of MMPs relies on Integrin activation [41]. In fact, Integrins cooperate with MMPs to promote breast cancer cell migration and invasion [42]. We predict that Nisch indirectly affects MMP expression. When Nisch reduces Integrin expression, this leads to a downstream reduction in MMP expression. Therefore, Nischarin positive breast cancer cells have a limited ability to degrade the ECM because they have decreased expression of MMPs. Taken together; our data show that the scaffolding protein Nisch regulates cell attachment and invadopodia formation by regulating the expression of numerous proteins. These data therefore demonstrate the power of Nischarin as a tumor suppressor, and its importance in preventing cell invasion.

\section{Conclusions}

In summary, we confirm our previous findings that Nischarin decreases cancer cell migration, and this is matrix independent. We then show that Nischarin alters the expression of other Integrins and multiple FA proteins. We further demonstrate the ability of Nisch to decrease invadopodia formation by altering the expression of some key invadopodia proteins. Since Nischarin is typically down regulated in cancer cells, these results help us understand why Nischarin-expressing cells have a slow migration rate.

\section{Additional file}

Additional file 1: Figure S1. A) WT and Null MEFs were seeded onto Fibronectin coated coverslip $(10 \mu \mathrm{g} / \mathrm{ml})$ overnight then stained with VASP. \# FA per cell and \% area covered by FA was acquired using CellProfiler. B) Co-Immunoprecipitation of Myc-Nischarin and VASP in HEK293 cells. (PDF 190 kb)

Abbreviations

ECM: Extracellular matrix; FA: Focal adhesion; MEF: Mouse embryonic fibroblast; MMP: Matrix metalloproteinase; PCR: Polymerase chain reaction

\section{Acknowledgements}

We would like to thank Edna Cukierman (Fox Chase Cancer Center) for her advice on manufacturing FDMs. We would also like to thank Kenneth Yamada (NIDCR) for the SNAKA51 antibodies.

\section{Funding}

This work is supported by Fred G. Brazda foundation and LSUHSC School of Medicine

\section{Availability of data and materials}

All published data and material are available upon request to the corresponding author

\section{Authors' contributions}

MM- planning experiments, acquisition, analysis, and interpretation of data, drafting the manuscript; SD- acquisition and analysis of data; SB- acquisition of data; SK-conception and study design, final approval of the manuscript; All authors read and approved the final manuscript.

Ethics approval and consent to participate

Not applicable.

Consent for publication

Not applicable.

\section{Competing interests}

The authors declare they have no competing interests.

\section{Publisher's Note}

Springer Nature remains neutral with regard to jurisdictional claims in published maps and institutional affiliations.

\section{Author details}

${ }^{1}$ Department of Biochemistry and Molecular Biology, LSUHSC School of Medicine, New Orleans, LA 70112, USA. ²Department of Biochemistry and Microbial Science, Central University of Punjab, Bathinda 151001, India.

Received: 9 January 2018 Accepted: 11 January 2018

Published online: 07 February 2018

\section{References}

1. Weidner N, Semple JP, Welch WR, Folkman J. Tumor angiogenesis and metastasis-correlation in invasive breast carcinoma. N Engl J Med. 1991;324:1-8. 
2. Aboussekhra A. Role of cancer-associated fibroblasts in breast cancer development and prognosis. Int J Dev Biol. 2011;55:841-9.

3. Jing Y, Han Z, Zhang S, Liu Y, Wei L. Epithelial-mesenchymal transition in tumor microenvironment. Cell Biosci. 2011;1:29.

4. Chaudhuri O, Koshy ST, Branco da Cunha C, Shin JW, Verbeke CS, Allison KH, Mooney DJ. Extracellular matrix stiffness and composition jointly regulate the induction of malignant phenotypes in mammary epithelium. Nat Mater. 2014;13:970-8

5. Schedin P, Keely PJ. Mammary gland ECM remodeling, stiffness, and mechanosignaling in normal development and tumor progression. Cold Spring Harb Perspect Biol. 2011;3:a003228.

6. Seewaldt V. ECM stiffness paves the way for tumor cells. Nat Med. 2014;20:332-3.

7. Cox TR, Erler JT. Remodeling and homeostasis of the extracellular matrix: implications for fibrotic diseases and cancer. Dis Model Mech. 2011;4:165-78.

8. Jinka R, Kapoor R, Sistla PG, Raj TA, Pande G: Alterations in cell-extracellular matrix interactions during progression of cancers. Int J Cell Biol 2012, 2012: 219196

9. Alahari SK, Nasrallah $\mathrm{H}$. A membrane proximal region of the integrin alpha5 subunit is important for its interaction with nischarin. Biochem J. 2004;377: 449-57.

10. Alahari SK. Nischarin inhibits Rac induced migration and invasion of epithelial cells by affecting signaling cascades involving PAK. Exp Cell Res. 2003;288:415-24.

11. Baranwal S, Wang Y, Rathinam R, Lee J, Jin L, McGoey R, Pylayeva Y, Giancotti F, Blobe GC, Alahari SK. Molecular characterization of the tumorsuppressive function of nischarin in breast cancer. J Natl Cancer Inst. 2011; 103:1513-28.

12. Ding Y, Milosavljevic T, Alahari SK. Nischarin inhibits LIM kinase to regulate cofilin phosphorylation and cell invasion. Mol Cell Biol. 2008;28:3742-56.

13. Jain P, Baranwal S, Dong S, Struckhoff AP, Worthylake RA, Alahari SK. Integrin-binding protein nischarin interacts with tumor suppressor liver kinase B1 (LKB1) to regulate cell migration of breast epithelial cells. J Biol Chem. 2013:288:15495-509.

14. Alahari SK, Reddig PJ, Juliano RL. The integrin-binding protein Nischarin regulates cell migration by inhibiting PAK. EMBO J. 2004;23:2777-88.

15. Maziveyi M, Alahari SK. Cell matrix adhesions in cancer: the proteins that form the glue. Oncotarget. 2017;

16. Deakin NO, Turner CE. Paxillin comes of age. J Cell Sci. 2008;121:2435-44.

17. Thomas SM, Hagel M, Turner CE. Characterization of a focal adhesion protein, hic-5, that shares extensive homology with paxillin. J Cell Sci. 1999; 112(Pt 2):181-90.

18. Tran TC, Singleton C, Fraley TS, Greenwood JA. Cysteine-rich protein 1 (CRP1) regulates actin filament bundling. BMC Cell Biol. 2005;6:45

19. Imbert AM, Garulli C, Choquet E, Koubi M, Aurrand-Lions M, Chabannon C. CD146 expression in human breast cancer cell lines induces phenotypic and functional changes observed in epithelial to mesenchymal transition. PLoS One. 2012;7:e43752.

20. Nix DA, Fradelizi J, Bockholt S, Menichi B, Louvard D, Friederich E, Beckerle MC. Targeting of zyxin to sites of actin membrane interaction and to the nucleus. J Biol Chem. 2001;276:34759-67.

21. Dong S, Baranwal S, Garcia A, Serrano-Gomez SJ, Eastlack S, Iwakuma T, Mercante D, Mauvais-Jarvis F, Alahari SK. Nischarin inhibition alters energy metabolism by activating AMP-activated protein kinase. J Biol Chem. 2017; 292:16833-46.

22. Gupton SL, Riquelme D, Hughes-Alford SK, Tadros J, Rudina SS, Hynes RO, Lauffenburger D, Gertler FB. Mena binds alpha5 integrin directly and modulates alpha5beta1 function. J Cell Biol. 2012;198:657-76.

23. Alahari SK, Lee JW, Juliano RL. Nischarin, a novel protein that interacts with the integrin alpha5 subunit and inhibits cell migration. J Cell Biol. 2000;151: $1141-54$.

24. Das AM, Eggermont AM, ten Hagen TL. A ring barrier-based migration assay to assess cell migration in vitro. Nat Protoc. 2015:10:904-15.

25. Dalby MJ, Yarwood SJ. Analysis of focal adhesions and cytoskeleton by custom microarray. Methods Mol Biol. 2007:370:121-34

26. McLean GW, Carragher NO, Avizienyte E, Evans J, Brunton VG, Frame MC The role of focal-adhesion kinase in cancer - a new therapeutic opportunity. Nat Rev Cancer. 2005;5:505-15.

27. Clark K, Pankov R, Travis MA, Askari JA, Mould AP, Craig SE, Newham P, Yamada KM, Humphries MJ. A specific alpha5beta1-integrin conformation promotes directional integrin translocation and fibronectin matrix formation. J Cell Sci. 2005;118:291-300.

28. Leong HS, Robertson AE, Stoletov K, Leith SJ, Chin CA, Chien AE, Hague MN, Ablack A, Carmine-Simmen K, VA MP, et al. Invadopodia are required for cancer cell extravasation and are a therapeutic target for metastasis. Cell Rep. 2014;8:1558-70

29. Artym W, Yamada KM, Mueller SC. ECM degradation assays for analyzing local cell invasion. Methods Mol Biol. 2009;522:211-9.

30. Clark ES, Weaver AM. A new role for cortactin in invadopodia: regulation of protease secretion. Eur J Cell Biol. 2008;87:581-90.

31. Courtneidge SA. Cell migration and invasion in human disease: the Tks adaptor proteins. Biochem Soc Trans. 2012;40:129-32.

32. Crimaldi L, Courtneidge SA, Gimona M. Tks5 recruits AFAP-110, p190RhoGAP, and cortactin for podosome formation. Exp Cell Res. 2009;315 2581-92.

33. Kohrmann A, Kammerer U, Kapp M, Dietl J, Anacker J. Expression of matrix metalloproteinases (MMPs) in primary human breast cancer and breast cancer cell lines: new findings and review of the literature. BMC Cancer. 2009;9:188.

34. Serrano-Gomez SJ, Maziveyi M, Alahari SK. Regulation of epithelialmesenchymal transition through epigenetic and post-translational modifications. Mol Cancer. 2016;15:18.

35. Chang C, Wei W, Han D, Meng J, Zhu F, Xiao Y, Wu G, Shi X, Zhang L. Expression of Nischarin negatively correlates with estrogen receptor and alters apoptosis, migration and invasion in human breast cancer. Biochem Biophys Res Commun. 2017:484:536-42.

36. Rangarajan R, Zaman MH. Modeling cell migration in 3D: status and challenges. Cell Adhes Migr. 2008;2:106-9.

37. Nagano M, Hoshino D, Koshikawa N, Akizawa T, Seiki M. Turnover of focal adhesions and cancer cell migration. Int J Cell Biol. 2012;2012:310616.

38. Irby RB, Yeatman TJ. Role of Src expression and activation in human cancer. Oncogene. 2000;19:5636-42.

39. Mueller SC, Ghersi G, Akiyama SK, Sang QX, Howard L, Pineiro-Sanchez M, Nakahara $H$, Yeh $Y$, Chen WT. A novel protease-docking function of integrin at invadopodia. J Biol Chem. 1999;274:24947-52.

40. Lohmer LL, Kelley LC, Hagedorn EJ, Sherwood DR. Invadopodia and basement membrane invasion in vivo. Cell Adhes Migr. 2014;8:246-55.

41. DiPersio CM, Shao M, Di Costanzo L, Kreidberg JA, Hynes RO. Mouse keratinocytes immortalized with large $T$ antigen acquire alpha3beta1 integrin-dependent secretion of MMP-9/gelatinase B. J Cell Sci. 2000;113(Pt 16):2909-21.

42. Rolli M, Fransvea E, Pilch J, Saven A, Felding-Habermann B. Activated integrin alphavbeta3 cooperates with metalloproteinase MMP-9 in regulating migration of metastatic breast cancer cells. Proc Natl Acad Sci U S A. 2003;100:9482-7.

\section{Submit your next manuscript to BioMed Central and we will help you at every step:}

- We accept pre-submission inquiries

- Our selector tool helps you to find the most relevant journal

- We provide round the clock customer support

- Convenient online submission

- Thorough peer review

- Inclusion in PubMed and all major indexing services

- Maximum visibility for your research

Submit your manuscript at www.biomedcentral.com/submit 